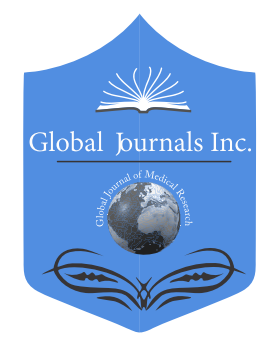

\title{
Baseline Data on Trend of Maternal Mortality in Tanzania using Administrative Data and its Policy Implication. 2018 Report
}

\author{
By Dr. Ahmad Mohamed Makuwani, Dr. Phineas Ferdinand Sospeter, \\ Dr. Leonard Subi, Dr. Mukome Anthony Nyamhagatta, Dr. Ntuli Kapologwe, \\ Mr. Habib Ismael, Dr. Naibu Mkongwa, Dr. Mpoki Mwasumbi Ulisubisya \\ \& Prof. Muhammad Bakari Kambi
}

Abstract- Background: Globally, Civil Registration, and Vital Statistics is the recommended method to track births and deaths. This system is weak in developing countries, including Tanzania. Other systems that may be used to report deaths, especially maternal mortality include integrated Disease Surveillance and Response (IDSR) and DHIS 2.

Tanzania has been using Demographic and Health Survey to track maternal deaths from as early as 2000. This study uses a sisterhood method which is conducted every five years, tracking events of the past ten years. It collects maternal deaths related from sisters of the same mother from sampled 10,000 households out of 11,000,000 available in Tanzania. The methodology uses wide confidence intervals, which affect its reliability. Therefore, the presented data is the outcome of tracking maternal deaths data using routine system from health facilities and communities in Tanzania Mainland.

GJMR-K Classification: NLMC Code: WA 310

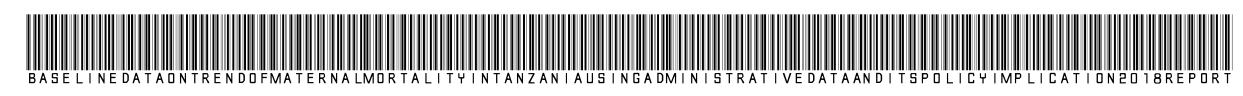

Strictly as per the compliance and regulations of:

(C) 2020. Dr. Ahmad Mohamed Makuwani, Dr. Phineas Ferdinand Sospeter, Dr. Leonard Subi, Dr. Mukome Anthony Nyamhagatta, Dr. Ntuli Kapologwe, Mr. Habib Ismael, Dr. Naibu Mkongwa, Dr. Mpoki Mwasumbi Ulisubisya \& Prof. Muhammad Bakari Kambi. This is a research/review paper, distributed under the terms of the Creative Commons Attribution-Noncommercial 3.0 Unported License http://creativecommons.org/licenses/by-nc/3.0/), permitting all non-commercial use, distribution, and reproduction in any medium, provided the original work is properly cited. 


\title{
Baseline Data on Trend of Maternal Mortality in Tanzania using Administrative Data and its Policy Implication. 2018 Report
}

\author{
Dr. Ahmad Mohamed Makuwani ${ }^{\alpha}$, Dr. Phineas Ferdinand Sospeter ${ }^{\sigma}$, Dr. Leonard Subi ${ }^{\rho}$, \\ Dr. Mukome Anthony Nyamhagatta ${ }^{\omega}$, Dr. Ntuli Kapologwe ${ }^{*}$, Mr. Habib Ismael $^{\S}$, Dr. Naibu Mkongwa ${ }^{\chi}$, \\ Dr. Mpoki Mwasumbi Ulisubisyav \& Prof. Muhammad Bakari Kambi ${ }^{\ominus}$
}

Abstract- Background: Globally, Civil Registration, and Vital Statistics is the recommended method to track births and deaths. This system is weak in developing countries, including Tanzania. Other systems that may be used to report deaths, especially maternal mortality include integrated Disease Surveillance and Response (IDSR) and DHIS 2.

Tanzania has been using Demographic and Health Survey to track maternal deaths from as early as 2000 . This study uses a sisterhood method which is conducted every five years, tracking events of the past ten years. It collects maternal deaths related from sisters of the same mother from sampled 10,000 households out of $11,000,000$ available in Tanzania. The methodology uses wide confidence intervals, which affect its reliability. Therefore, the presented data is the outcome of tracking maternal deaths data using routine system from health facilities and communities in Tanzania Mainland.

Methodology: Data collected were from all regions, all health facilities, and communities in Tanzania Mainland using National Governance structures on a daily basis. No funds were used to collect data except for airtime to make calls and internet connectivity.

Results: A total of 1,744 maternal deaths were mapped from health facilities and community. This number was adjusted for abortion $5 \%$, ectopic pregnancy $5 \%$, community deaths $10 \%$, and unreported $5 \%$. The final adjusted number of maternal deaths was 2,138 . Through a regional comparison of maternal deaths with population density, regional variation was critically noted. The data showed that $70 \%$ of maternal deaths were caused by PPH (29\%), Eclampsia (18.9\%), Anemia ( $8.8 \%$, puerperal sepsis $(7.9 \%)$, and suspected venous thromboembolism (5.5\%). Anesthetic complication contributed to $3.3 \%$ of all maternal deaths.

Conc/usion: Tanzania's health sector is mature enough to use routine data on maternal deaths to inform policy. However, a comprehensive, rigorous study needs to be conducted in Tanzania to come up with a better methodology of estimating maternal mortality ratio using routine data.

Author $\alpha \sigma \rho \omega \S \chi \theta$ : Ministry of Health, Community Development, Gender, Elderly and Children.e-mail: ahmadi.makuwani@afya.go.tz Author ¥: Presidents Office, Regional Administration and Local Government.

Author v: Former Permanent Secretary of the Ministry of Health, Community Development, Elderly and Children.

\section{Literature Review}

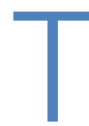
he Millennium Development Goals (MDGs) launched in 2000 required all United Nations member states to be committed to increase investments to improve the life of people worldwide. Goal number 5 was among the goals that needed member states to reduce the magnitude of maternal mortality by $75 \%$. Tanzania is one of the members in the global community; she committed herself to meet the goal above by reducing maternal mortality ratio to 193 deaths per 100,000 live births by 2015. To monitor progress, the DHS study was conducted in 2005,2010 , and 2015, and these showed that maternal mortality ratio was 578 per 100,000 live births, 454 per 100,000 live births, and 556 per 100,000 live births, respectively $(1,2,3)$.

Tanzania, in the last two decades, has invested heavily in socioeconomic development, especially in health, by increasing modern contraceptive use among women of reproductive age from $7 \%$ (1990) to 32\% (2016) and 38\% (2019). Furthermore, there was an increase in the health work force to almost 52\% (2018), and EmONC services availability was 32\% in 2015. TDHS 2016 report shows that the proportion of women attending the fourth antenatal clinic was $51 \%$, delivery conducted by skilled birth attendants was $64 \%$, and RMNCAH scorecard (January-March 2019) showed that postpartum attendance was $78 \%(3,4)$. Furthermore, Tanzania enjoyed, an increase in political commitment for health shown by Tanzania becoming a co-chair of UN commission on Information and accountability for women and children (2010/2011), launch of Tanzania Sharpened One Plan (2014-2015) and a significant increase in resource allocation in health systems that commenced from 2016 by the new Government led by His Excellency Dr. John Pombe Joseph Magufuli, the 5th President of United Republic of Tanzania $(5,6)$. The Lancet countdown report to 2015 observed that although Tanzania between 1990 - 2015 doubled the population and total budget allocated to the health sector, and increased three-times donor funding for child health and HIV/AIDS interventions, these 
increments did not correlate with decline in maternal and newborn deaths (7).

From 1990, Tanzania has been tracking data on maternal process indicators and outcome by relying on surveys, demographic and health surveys (DHS), and population census. The DHS developed in late 1980 use sisterhood methodology whose questions targets adult sisters from the same mother enquiring about the outcome of fertility history of female siblings. The method is highly limited due to the use of a small sample size aiming at reducing cost. For example, DHS conducted in Tanzania 10,000 households out of $11,000,000$ households have been traditionally being selected. Understanding its limitation, WHO recommends using this methodology for a population with a high fertility rate as it is insensitive to capture maternal deaths when Total Fertility Rate (TFR) is below 3 (8). The major disadvantage of this methodology is because the result emanating from the sisterhood method relates to a point around 10-12 years. As a result the method cannot provide a current estimates for the year of the survey hence making it neither useful to monitor changes in maternal mortality nor to assess the impact of safe motherhood programs in the short term (3). Mgawadere, et al. (2017) echoed the guidance by WHO by showing that Population or household surveys may be critically important to inform the situation on maternal mortality in countries where routine information systems are weak or non-existent (9).

\section{RATIONALE}

Tanzania has been investing heavily with high political commitment stewardship in improving health, especially RMNCAH services in areas of family planning, skilled birth attendants, EmONC services, and provision of enabling environment. There has been a big appreciable improvement in service delivery, as shown by an increase in users of modern contraceptive services, ANC-4 attendees, delivery conducted in the health facility and skilled birth attendant, and proportion of women receiving postpartum care $(1,2,3)$.

Improvement in the Tanzania transport system has a significant contribution in reducing the second level delay of access to receive health care services. The availability of good roads and means of transport facilities is critical in achieving this goal. Tanzania Road Authority (TANROADS) in 2018 reported that 67.2\% $(8,211 \mathrm{KM})$ of the roads in Tanzania are paved with almost all regions and the majority of districts connected to this network hence compressing the catchment population between these roads (10).

Significant development has also been made in improving available means of transport that range from vehicles, good buses, and motorcycles. The increase in the motorcycle population in East Africa, so as Tanzania, has made a noticeable difference in the transport of critically ill patients, pregnant women during labor and complications by reducing the time taken, from the hard to reach areas to a service delivery point of care. Chen et al. (2017) in Kigoma region showed that motorcycles have made a big difference towards the improvement of access of EmONC health facilities. The report showed that $13 \%$ of live births took place in areas where women were able to access health facilities within 2 hours on foot and 33\% in areas where motorized vehicles, including motorcycle, were needed to reach the EmONC health facility within 2 hours (11). Save the Children in Kenya (2016) showed testimony from women and community that motorcycles were beneficial in making sure women in need reached in time to a nearby EmONC health facility just in time receive life-saving services for themselves and that of the newborn (12). Schmitz et al. (2019) in Uganda demonstrated that the proportion of women of reproductive age accessing care within 2 hours from 2016 by motorcycles took an upward trend for a facility that provides any EmONC services and comprehensive EmONC services from $61 \%$ to $72 \%$, and $51 \%$ to $70 \%$, respectively (13).

Literacy is a key in gender dynamics towards having liberty in deciding where to seek care, that determine survival and well-being along a continuum of women cycle from pre-pregnancy, antenatal, labor and delivery, and postnatal care. Tanzania 2019 report by UNESCO showed that there is an increase in literacy among girls aged $15-24$ years from $78 \%$ in 1990 to $85 \%$ in 2015, making more girls accessing more information to meet their need in sexual reproductive health (14).

From 2015 the health sector has been following up the maternal outcome data using routine data. The language at the national and global levels on the number of MD has always been higher at around 11,000 deaths per year as reported by TDHS 2016. These numbers contradicted those reported by regions, which stands at lower (3). In essence, the situation was like the national and global levels were forcing regions to accept the numbers, which are imposed on them. For this reason, the Government of Tanzania, in 2018, undertook a decision to increase stewardship to follow up MD using MPDSR guideline by using national governance structure after understanding that the use of CVR, DHIS2, and IDSR was very low (15).

Therefore this baseline report aimed to use available routine data to:

1. Assess and ascertain the real-time situation of $M D$ in Tanzania using administrative data.

2. Analyze causes of MD to inform policy interventions.

3. Advise policy on best approaches to use in maternal data collection. 


\section{Methodology}

This report describes the process of counting MD in Tanzania conducted in January - December 2018 in line with Every Woman Every Child Initiative.

Using a strong Governance structure available in Tanzania, MD data collected were from all health facilities, communities, districts, and regions.

a) Setting the ground for data collection

In 2015 the National MPDSR Guideline was ratified to be used in Tanzania Mainland in line with WHO and FIGO recommendations. The following year (2016), the Guideline was disseminated to Regional Health Management Teams (RHMTs), Council Health Management Teams (CHMTs), and Health Facilities Management Teams (HFMTs) of all the 26 regions. The Guideline requires notification for every maternal death that has occurred from the health facility and community settings within 48 hours. It also directs to the review of MD within seven days by the health facility maternal death review committee. Furthermore, the committee is required to regularly convene after every notified maternal death or after the occurrence of a near-miss case or monthly when there is no reported maternal death (15).

Similarly, the Guideline directs the formation of a district council, which meets monthly and regional MD review committee, meeting after every three months. The Ministry responsible for Health further appointed a National Technical Committee for Maternal Death Reviews, which is supposed to meet bi-annual to receive and discuss MD regional reports, make analysis, provide recommendations and sometimes effect change in various guidelines in practice based on recommendations (15).

Aligning to WHO guidance through a 2004 guideline (Beyond the numbers), the process of maternal death review follows a principle of strict confidentiality that information emanating at each level should not leak to outsiders, and all information used and collected should be kept under lock and key. Confidentiality leads to openness in describing causes and factors leading to adverse maternal outcomes. The guideline goes further to emphasize that the process should be anonymous, done under a non-threatening environment, and never the review process should be means of apportioning blame or provide a basis for litigation or management sanctions $(15,16)$.

In 2018, the Ministry urged local data experts to discuss a possibility of using routine data to inform estimation of maternal and U5 deaths. Experts noted that in Tanzania, Civil Registration and Vital Statistics (CRVS), DHIS-2 and Integrated Disease Surveillance System (IDSR) were not strong enough to track maternal and under-five deaths. Also, experts acknowledge that Tanzania has a solid governance structure that may be improvised and used to record/track health facility and community MD. Likewise, both health facilities and community were observed to have parallel systems and tracking tools for all deaths. The expert review further identified that Urassa et al. (1994) a study from Muhimbili University of Health and Allied Sciences (MUHAS), showed that only $10 \%$ of all MD, reported in Ilala district, occurred in community setting and the rest were from within health system (17).

\section{Data Collectors}

Zonal, Regional and District Reproductive and Child Health Coordinators ( $\mathrm{RCHco}$ ) were the strategic officers responsible for January - December 2018 maternal death data collection. The data collection was in line with the National MPDSR Guidelines. The District $\mathrm{RCHCos}$ were directly involved in MD notification, reviews, and data collection from health facilities that include dispensaries, health centers, and hospitals, and community within the district council. The collected data from the district council were submitted on a weekly basis to Regional and Zonal RCHCos, who finally transmitted it to the National level by using a designated MPDSR email address for only data collection.

\section{a) Data Collection Tool}

A standardized maternal death data collection tool was used to collect the following individual variables; reporting region and district, health facility or community, date, age, gravity and parity, and clinical cause of death. This tool captured suspected MD notified and submitted to the Regional Medical Officer whose duty was to approve before submitting to the Ministry of Health at Reproductive and Child Health Section. As per MPDSR Guideline, all reported MD had to be reviewed within 7-days of occurrence and categorized to ICDM 10 classification at health facility supported by district and regional experts (15).

Notified data of MDs from regions were consolidated into the Excel spreadsheet, shared, verified, and validated by regions on a monthly, quarterly, and semi-annual basis after the review. The collected maternal death data were disseminated on a weekly basis at the Department of Preventive Services, quarter and semi-annual at $\mathrm{MOH}$ management. Final data were disseminated to different levels and various platforms, including scientific conferences, Government, and stakeholders meeting. Through an official letter signed by Permanent Secretary, and the final MD report was submitted officially to the National Bureau of Statistics.

\section{b) Data quality control}

To ensure data quality, the District Reproductive and Child Health Coordinator made active surveillance and the follow up of any reported probable-suspected or confirmed maternal death from a health facility or 
community setting. For reported suspected community maternal death, a team of district Maternal Death Review Team participated in the funeral and later after about a week returned to conduct a verbal autopsy. Furthermore, the Regional team implemented quarterly supportive supervision to hospitals and health centers where they did verification and validation of reported MD.

c) Controlling the source of error in calculating the total number of $M D$

The maternal death data collected are required to be representative of the actual situation in the Country hence a need for employing some statistical assumption. In Tanzania, the current institution delivery is $80 \%$, and the postnatal visit is around $78 \%$ (18). Mgawadere et al. (2017) report noted that to use health facility data to estimate the reliable magnitude of maternal mortality, institution delivery should be more than $85 \%$ (9). In approximating total MD using any method, the biggest challenge is to account for ectopic pregnancies and abortions, reported to contribute 5\% and $8 \%$ of MD, respectively $(19,20,21)$ (Box 1$)$.

Box 1: Assumption of possible missed MD (MD)

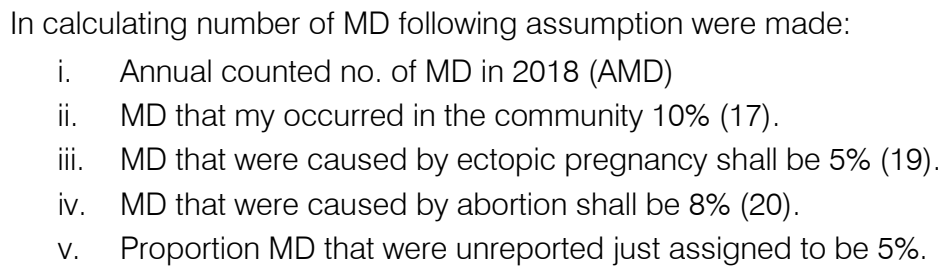

From the assumption above the actual collected maternal deaths were adjusted accordingly.

Box 2: Adjusted MD

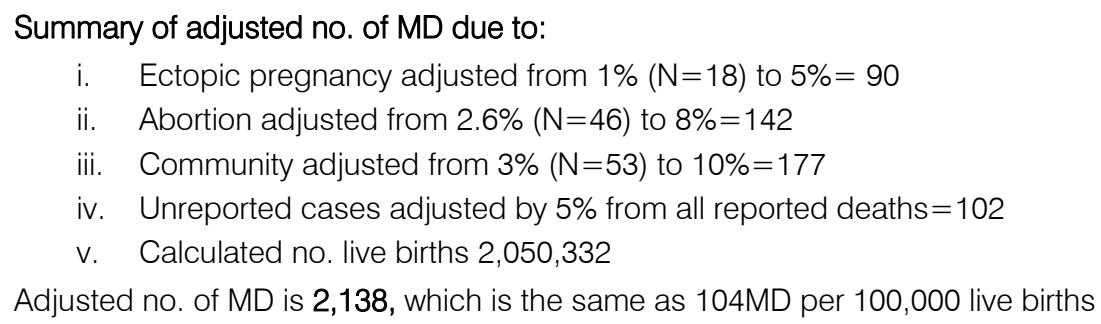

\section{d) Evaluation strategy}

In this documentation, the intention is to demonstrate the implication of follow up of $\mathrm{MD}$ conducted in 2018 and an ongoing process in monitoring and evaluation of RMNCAH interventions by measuring the outcome. The presentation of data will base on reporting simple ratios.

\section{Results}

These data were collected from both health facilities and community, and 639 health facilities provided data on MD. These health facilities were both national referral one national hospital (1), three zonal and 28 regional referral hospital and district hospitals, health centers, and dispensaries. The number provided makes $100 \%$ coverage of all health facilities which reported MD in 2018. Also, 3\% of reported MD occurred in the community. In this report, all 185 district councils had at least one maternal death; none of the districts had zero MD for the entire year.

Generally, the total number of MD was 1,744 , with an indication that the majority of MD occurring in the health facilities as only 53 (3\%) reported from the community.

Using the assumption that the cause of maternal death by ectopic pregnancy is $5 \%$, abortion $8 \%$, unreported $5 \%$, and community $10 \%$. Recalculated estimated number of MD was 2,138, a count that is within the earlier expected number of $\mathrm{MD}$ that range from 1,500 to as high as $2,500 \mathrm{MD}$.

\section{a) Calculated Maternal Mortality Ratio}

The analysis went further to calculate maternal mortality ratio (MMR) based on the projected number of MD as per regional and national levels. The data showed that the estimated National maternal mortality ratio was 104 deaths per 100,000 live births with 11 regions standing above the national average, which are led by Dar Es Salaam with MMR of 221 MD per 100,000 live births and Tabora region had the lowest MMR with 52 deaths per 100,000 live births below the national average. 


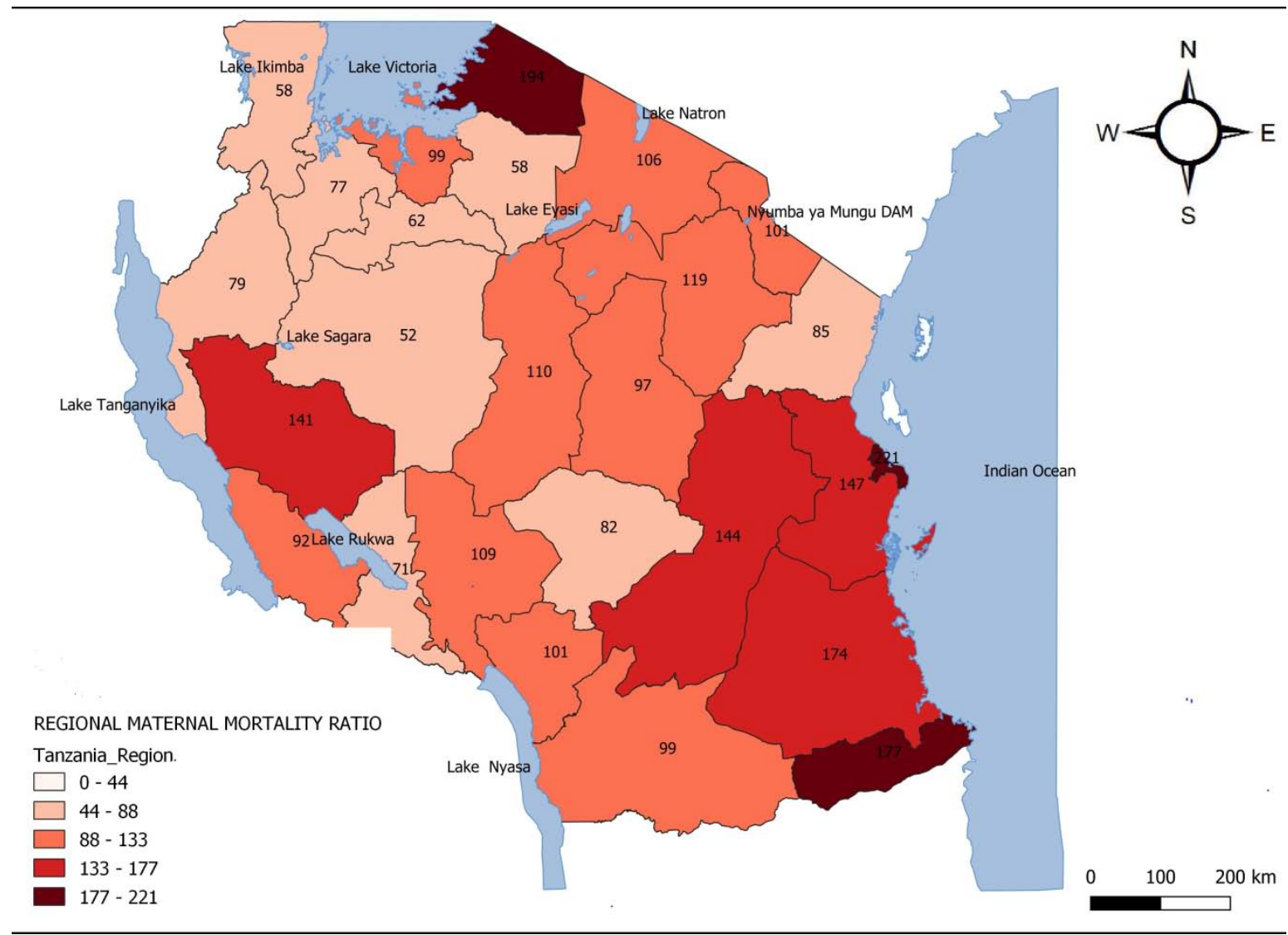

Figure 1: Spatial Mapping of MMR in Tanzania Using Administrative Data of 2018

\section{b) Distribution of $M D$}

MD data in 2018 were not equitably distributed across the country with the distribution showing that Dar Es Salaam, Mwanza and Morogoro regions were the top three regions with the highest number of deaths accounting for $26.4 \%(\mathrm{~N}=462)$ of the burden, while Songwe, Manyara and Njombe regions were taking the tail, contributing only $4.8 \%(\mathrm{~N}=84)$. A different picture was observed when the number of MD was weighed against the total population in the region. Five regions, namely Katavi, Mtwara, Pwani, Lindi and Morogoro regions had the highest weight with 8.2, 5.7, 5.5, 5.3 and 5.1 MD per 100,000 population per year, respectively, and Njombe region showed to have the lowest weight per population, which was 1.5 MD per 100,000 population per year. In this analysis showed that, although Katavi region has the smallest population of 650,000 people compared to other regions, this region had the biggest weight per population of $8.2 \mathrm{MD}$ per 100,000 population than Dar Es Salaam region with the biggest population of 5.5 million people with the least weight per population of 3.4 MD per 100,000 population.

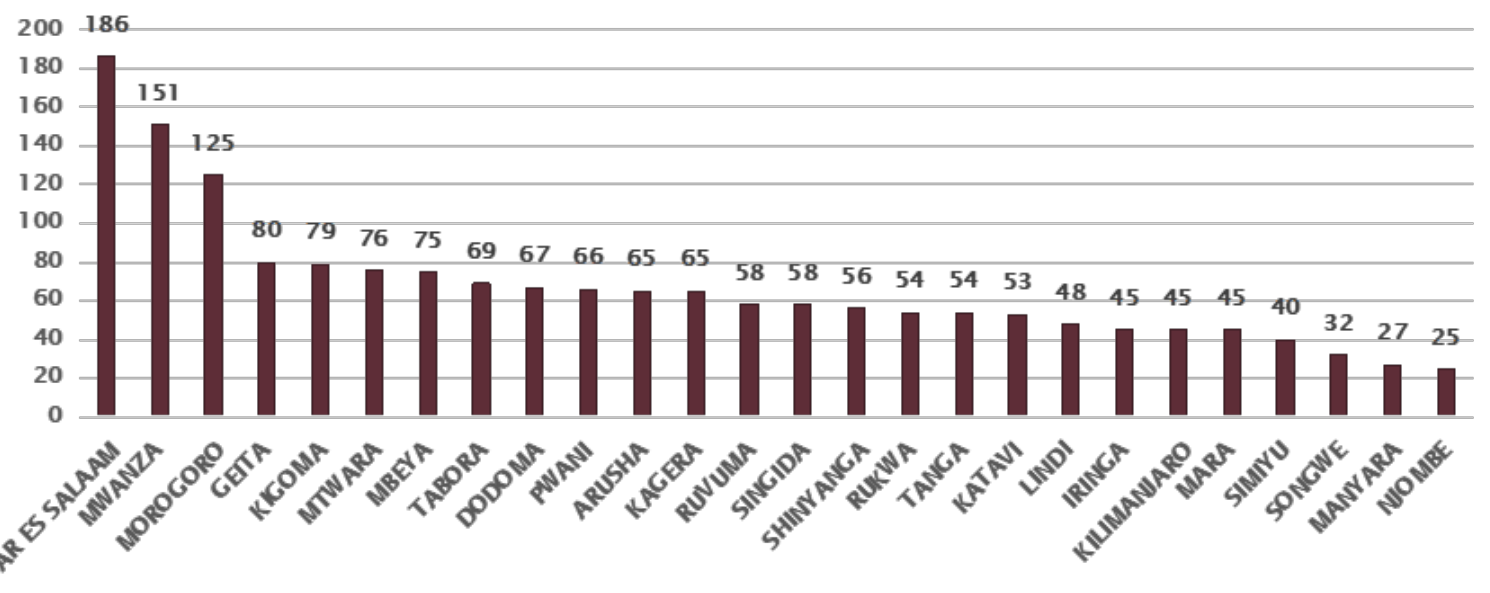

Figure 2: Absolute (actual) No. of MD before adjustment per region $(\mathrm{N}=1,744)$ 


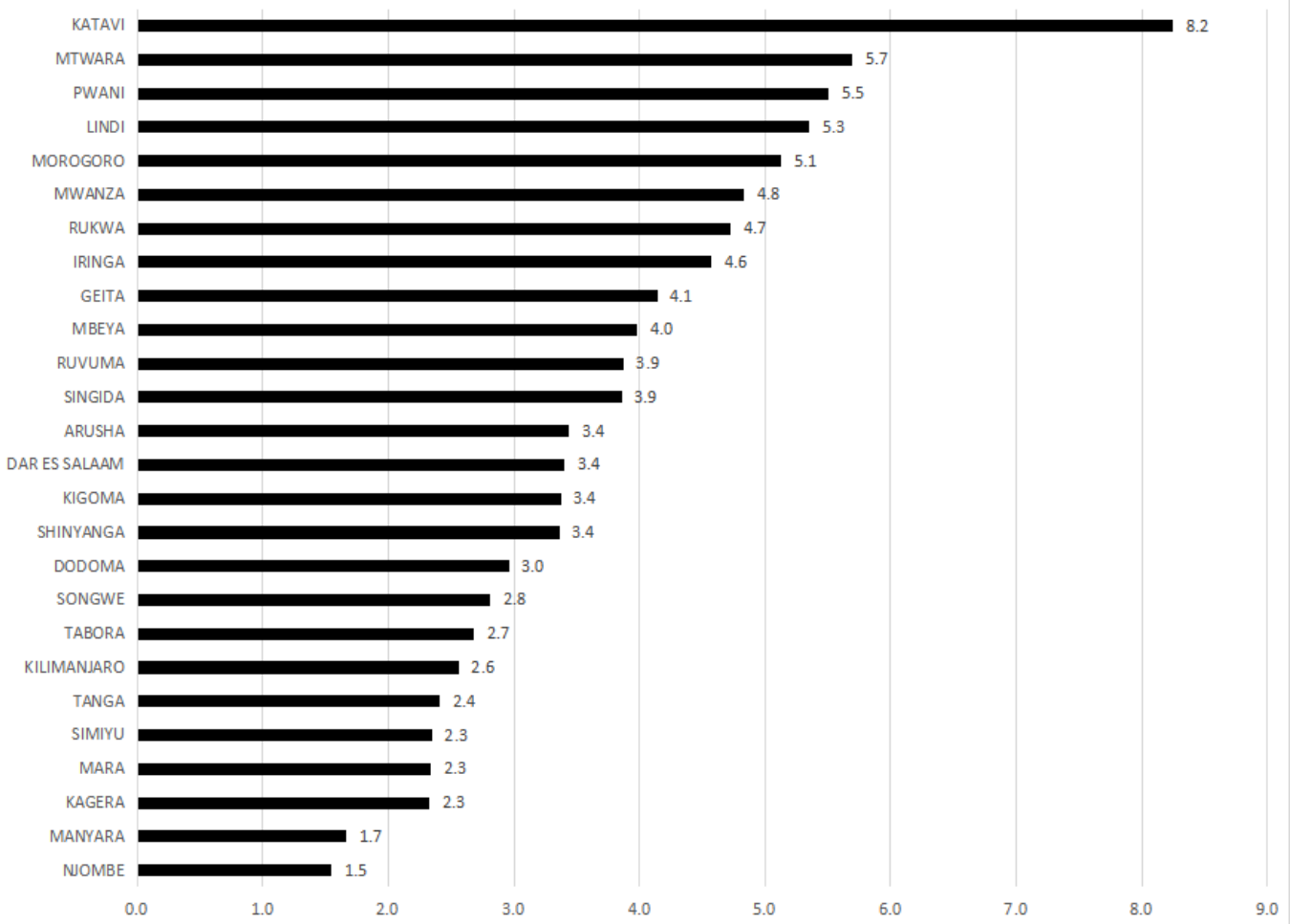

Figure 3: Weighted No. of MD per population

\section{c) Cause of Maternal Deaths}

Data reported in 2018 on MD provides enormous information regarding where and what caused MD, and this was possible through MD reviews conducted using a National MPDSR guideline at the health facility, district council, regional and national level. Though in Tanzania, the sisterhood method had been the source of estimation of maternal mortality ratio, the methodology consistently lacked to provide critical analysis on the cause and where MD occurred. The
2018 report on maternal mortality showed that $70 \%$ of deaths were caused by mainly five major complications, which were Postpartum hemorrhage $(29.0 \%, N=506)$, Eclampsia related complication (18.9\%, $\mathrm{N}=329)$, Severe anemia in pregnancy $(8.8 \%, N=154)$, obstetric sepsis (7.9\%, $\quad \mathrm{N}=139)$ and suspected venous thromboembolism $(5.5 \%, \mathrm{~N}=96)$ and $32 \%$ of deaths occurred in referral hospitals, which are regional, zonal and national referral hospitals.

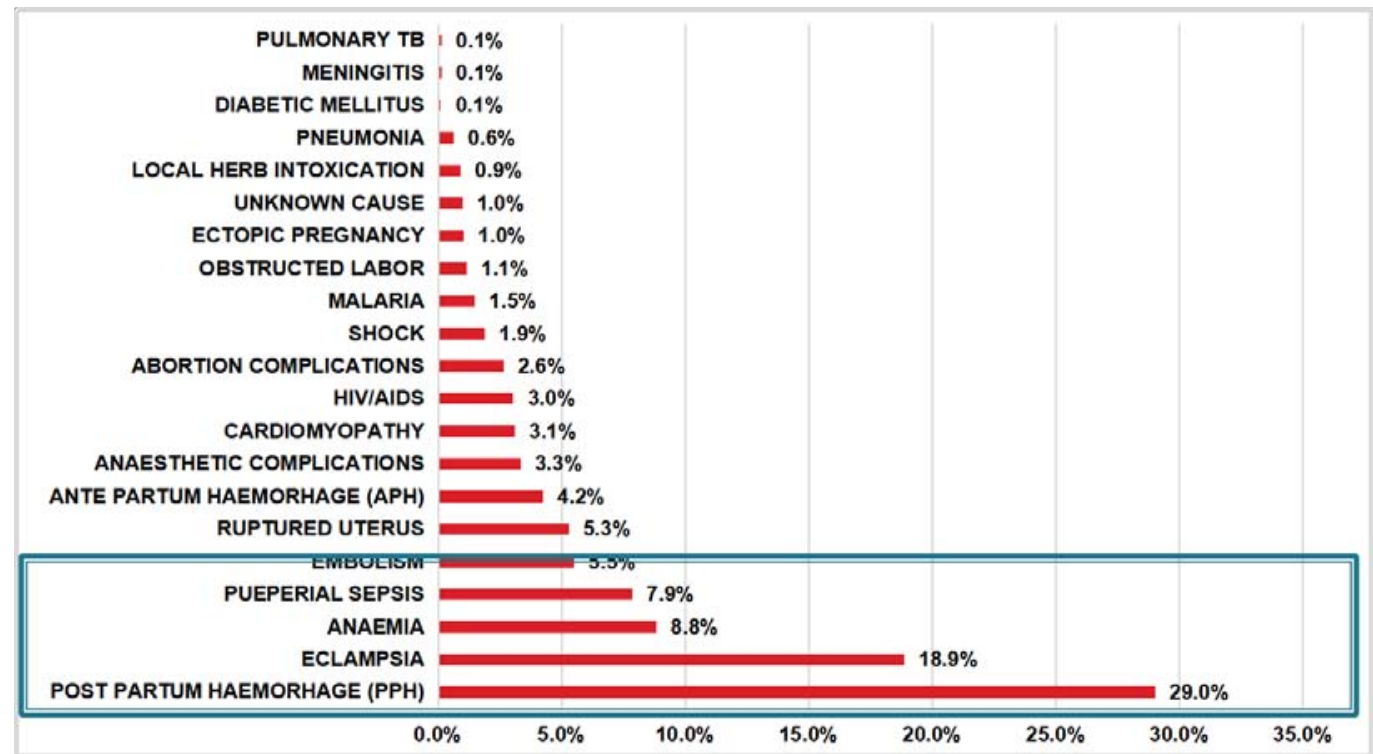

Figure 4: Causes of MD as classified using ICD $10(\mathrm{~N}=1,744)$ 


\section{Discussion}

Commencing from 2016 - 2019 Tanzania took seriously tracking and use of routine maternal, newborn, and child deaths data in programming and measuring progress. The MD registration has been possible by the use of the strong Country Governance Structure that has enabled to track data on Maternal, Newborn, and Child Health outcome from both health facilities and community. Implementation of MPDSR Guidelines has been a useful tool in the notification and classification of MD. As reported in the previous section, data collection was, and continue to be conducted manually from health facilities and community to district council via District Reproductive and Child Health Coordinators, and at Region level by Regional Reproductive and Child Health Coordinators and finally to the Ministry of Health, Community Development, Gender, Elderly and Children at the Reproductive and Child Health Section. This paper negates the prevailing assumption that that the Country health system cannot track the maternal, newborn, and child health outcome data. Waiswa et al. (2019) observed that under-reporting of pregnancies and birth outcomes is a major issue that needs to be addressed (21). In the case of Tanzania, the development, dissemination, and use of MPDSR Guideline has enabled to great extent to mitigate this challenge of under-reporting.

This report observed that following the adjustment of data using the assumption, the calculated National MMR was 104 deaths per 100, 000 live births with a regional range of as high as 220 deaths per 100,000 live births for Dar Es Salaam Region to as low as 54 deaths per 100,000 live births for Tabora Region. A CDC report tracking decline maternal mortality in Kigoma in 2018 showed that maternal mortality ratio for the Region was 174 deaths per 100,000 live births, twice as much to the magnitude presented in this paper, which is 82 deaths per 100,000 live births. The variation above may be attributed to the methodology used by the later, whose data collection was confined to the health facilities to monitor progress EmONC intervention (22).

The report further shows that when the number of $\mathrm{MD}$ is compared with a regional population, a different picture is displayed. Katavi population was leading with the bigger weight of MD per population of 8.2 deaths per 100,000 people, followed by the Mtwara region with 5.7 deaths per 100,000 population. The region which had the least weight was Njombe Region, with 1.5 deaths per 100,000 live births.

Unlike the sisterhood method, which can provide a country MMR for Global level advocacy, the use of routine data carries more information to inform policy and implementation. This paper demonstrated that the top five leading causes of all $70 \%$ of MD were postpartum hemorrhage, eclampsia, anemia, sepsis, and suspected thromboembolism. The observation above tally with that of Mgawadere et al. (2017), which observed that health facility MD data in many developing countries provide valuable information on where and why maternal death occurred hence calling for a need to invest in them (9).

\section{Vil. ReCOMmEndATION}

Published data demonstrate the value of using health facility data to describe the burden of MD beyond the numbers aligned to Every Woman, Every Child (EWEC) initiative. Finally, it fair to recommend that Tanzania and possibly to other Sub Saharan Countries that the health sector is mature enough to use routine data on maternal deaths to inform policy. However, a comprehensive, rigorous study needs to be conducted to come up with a better methodology of estimate MMR using routine data.

\section{AcKnowledgement}

Authors would like to express sincere gratitude to all key players of implementation of MPDSR guidelines in Tanzania by making availability of routine MD data in the country. Special thanks should go to all RHMT, CHMT, Regional and District Medical Officers, ZRCHCo, RRCHCo, DRCHCO, Health Facility Management Teams, and RMNCAH implementing partners for working hard to make findings above a reality. Last but not least we thank the Ministry of Health, Community Development, Gender, Elderly and Children team - Department of Preventive Services, Reproductive Health Section for stewardship of MD and other routine data collection.

\section{References Références Referencias}

1. National Bureau of Statistics (NBS) [Tanzania] and ORC Macro. 2005. Tanzania Demographic and Health Survey 2004-05. Dar es Salaam, Tanzania: National Bureau of Statistics and ORC Macro.

2. National Bureau of Statistics (NBS) [Tanzania] and ICF Macro. 2011. Tanzania Demographic and Health Survey 2010. Dar es Salaam, Tanzania: NBS and ICF Macro.

3. Ministry of Health, Community Development, Gender, Elderly and Children (MoHCDGEC) [Tanzania Mainland], Ministry of Health $(\mathrm{MoH})$ [Zanzibar], National Bureau of Statistics (NBS), Office of the Chief Government Statistician (OCGS), and ICF. 2016. Tanzania Demographic and Health Survey and Malaria Indicator Survey (TDHS-MIS) 2015-16. Dares Salaam, Tanzania, and Rockville, Maryland, USA: MoHCDGEC, MoH, NBS, OCGS, and ICF.

4. Ministry of Health and Social Welfare (United Republic of Tanzania), 2019. RMNCAH Scorecard, January - March, 2019 Report. 
5. World Health Organization 2011. Commission on information and accountability for Women's and Children's Health. Keeping promises, measuring results. Final report of the Commission.

6. Ministry of Health and Social Welfare (United Republic of Tanzania), 2014. Sharpened One Plan 2014-2015 for The National Road Map Strategic Plan to Accelerate Reduction of Maternal, Newborn and Child Deaths in Tanzania 2008-2015.

7. Afnan-Holmes $H$, Magoma $M$, John $T$, Levira $F$, Msemo G, Armstrong CE, et al. Tanzania's countdown to 2015: an analysis of two decades of progress and gaps for reproductive, maternal, newborn, and child health, to inform priorities for post-2015. Lancet Glob Health. 2015; 3: e396-409.

8. World Health Organization, 1997. The sisterhood method for estimating maternal mortality: Guidance notes for potential users.

9. Mgawadere F, Kana T, Nynke van den Broek. Measuring maternal mortality: a systematic review of methods used to obtain estimates of the maternal mortality ratio (MMR) in low and middle income countries. Br Med Bull. 2017;122:1. doi: 10.1093.

10. Ministry of Works, Transport and Communications (United Republic of Tanzania), TANROAD, 2018. Trunk and Regional Roads Network.

11. Chen YN, Schmitz MM, Serbanescu F, Dynes MM, Maro G, Kramer MR. Geographic Access Modelling of Emergency Obstetric and Neonatal Care in Kigoma Region, Tanzania: Transportation Schemes and Programmatic Implications. Glob Health Sci Pract. 2017; 5:430-445.

12. Save the Children, 2016. Children from minority groups Kenya, Boda Boda' ambulances...Simple and lifesaving for mothers and children.

13. Schmitz MM, Serbanescu F, Kamara V, Kraft JM, Cunningham M, Opio G, et al. Did Saving Mothers, Giving Life Expand Timely Access to Lifesaving Care in Uganda? A Spatial District-Level Analysis of Travel Time to Emergency Obstetric and Newborn Care. Glob Health Sci Pract. 2019; 7: S151-S167.

14. UNESCO Institute of Statistics, 2019. United Republic of Tanzania, Sustainable Development Goals. Education and Literacy.

15. Ministry of Health and Social Welfare (United Republic of Tanzania), 2015. Maternal And Perinatal Death Surveillance And Response Guideline.

16. World Health Organization, 2004. Beyond the numbers: reviewing maternal deaths and complications to make pregnancy safer.

17. Urassa E, Massawe S, Mgaya H, Lindmark G, Nyström L. Female mortality in reproductive ages in Dar es Salaam, Tanzania. East Afr Med J. 1994; 71 : 226-31.

18. Ministry of Health, Community Development, Gender, Elderly and Children (United Republic of Tanzania) 2019. Reproductive, Maternal, Newborn,
Child and Adolescent Health (RMNCAH) Scorecard Q1, January - March, 2019.

19. Kamga DVT, Nana PN, Fouelifack FY, Fouedjio JH. Role of abortion and ectopic pregnancies in maternal mortality rate at three university hospitals in Yaoundé. Pan Afr Med J. 2017; 27:248.

20. Say L, Chou D, Gemmill A, Tunçalp Ö, Moller AB, Daniels J, Gülmezoglu AM2, Temmerman M, Alkema L. Global causes of maternal death: a WHO systematic analysis. Lancet Glob Health. 2014; 2: e323-33.

21. Waiswa P, Akuze J, Moyer C, Kwesiga D, Arthur S, Sankoh $\mathrm{O}$, et al; INDEPTH Network MNCH team. Status of birth and pregnancy outcome capture in Health Demographic Surveillance Sites in 13 countries. Int J Public Health. 2019; 64: 909 - 920.

22. U.S. Centers for Disease Control and Prevention. (2019). Reducing Maternal Mortality in Tanzania: 2019 Pregnancy Outcomes Study in Kigoma Region, Tanzania: Executive Summary. Atlanta, GA: Centers for Disease Control and Prevention. 